\title{
The introduction of powder blasting for sensor and microsystem applications
}

\author{
E. Belloy * , S. Thurre, E. Walckiers, A. Sayah, M.A.M Gijs \\ Institute of Microsystems, Swiss Federal Institute of Technology of Lausanne, EPFL, CH-1015 Lausanne, Switzerland
}

Received 3 August 1999; received in revised form 18 January 2000; accepted 24 January 2000

\begin{abstract}
We introduce powder blasting using a pressurised nozzle and a metallic mask as a new promising technology for microsystem fabrication. We study basic parameters of this powder blasting erosion process as well as mask-geometry effects on the erosion rate. We demonstrate the application potential of this technique in three important fields of microsystems research: (i) the realisation of microfluidic chips for biochemical separations, (ii) the micropatterning of composite hard magnetic layers for mechatronic and magnetic sensor applications, and (iii) the realisation of inertial sensors in glass. We present for the first time a mechanical and electrical characterisation of powder-blasted accelerometer devices. (C) 2000 Elsevier Science B.V. All rights reserved.
\end{abstract}

Keywords: Powder blasting; Microfabrication; Accelerometer; Erosion

\section{Introduction}

Traditionally, particle erosion is a cumbersome phenomenon to be avoided in a lot of applications. It occurs when solid particles contained in a high-speed air jet damage turbine rotor blades or motor parts. Erosion behaviour is affected by the particle type and the characteristics of the target material. Depending on the indenting particle type (blunt or sharp) and the target material (ductile or brittle) the erosion process is different [1]. Ductile metal erosion has been studied using both macroscopic hardened steel spheres [2] and microscopic particles [3]. Brittle materials, like ceramics and glass, have been mainly studied using the impact of hard and sharp eroding particles.

\subsection{Basics of the erosion process}

The erosion mechanism is shown to be very similar to what is known from quasi-static or scratching Vickers-in-

\footnotetext{
* Corresponding author. Tel.: +41-21-693-6759; fax: +41-21-6935950.

E-mail address: eric.belloy@epfl.ch (E. Belloy).
}

dentation measurements. When the high stress underneath the sharp indenting particle exceeds a certain fracture threshold, cracks develop leading to removal of part of the target material [4,5]. As demonstrated in Ref. [6], an important parameter in the erosion process is the total incoming kinetic energy of the powder particles. The erosion efficiency $E_{\text {eff }}$ was introduced, which is the ratio of the removed material weight to the kinetic energy of incoming particle flux. It was argued that $E_{\text {eff }}$ is a better parameter to characterise the erosion process than the erosion rate $E_{\text {rate }}$, which is defined by the ratio of removed material weight to the overall weight of impacting particles. Indeed, not only the particle mass itself is important but much more its kinetic energy associated impact for having an efficient erosion process.

When a powder particle indents the surface of a brittle material (e.g. glass or silicon) it generates cracks and a plastic zone around the impact point. These cracks are of two types: lateral and radial. The lateral cracks play a major role in the erosion process because they can reach the surface removing upper material zone. This phenomenon is the principal cause of material removal. The radial cracks have no significant contribution in the erosion process, but they weaken the material. The length of such cracks depends on a few parameters like the substrates Young modulus, its hardness and the indentation 


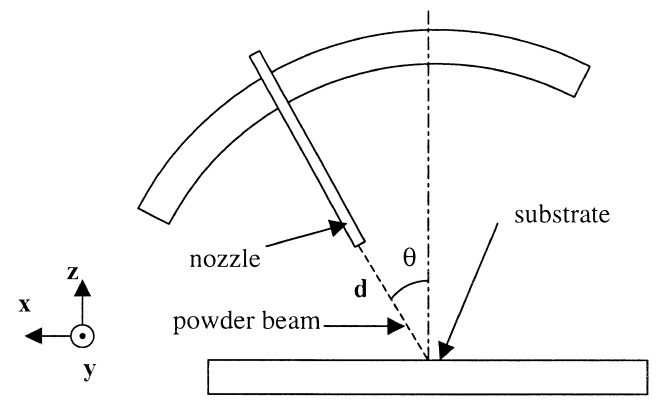

Fig. 1. Schematic view of the used powder-blasting system configuration.

volume. These parameters, in turn, depend both on the kinetic energy of the particle and its shape.

\subsection{Modern applications}

During recent years, the study of particle erosion processes has been a subject of increasing interest [1,7]. Powder blasting has been refined to reproducibly pattern large glass surfaces for flat panel display applications and was shown to be the most attractive and economically feasible for this kind of application [8-10]. The flat panel display application requires the reproducible realisation of millions of millimeter-sized holes on large (of order $1 \mathrm{~m}^{2}$ ) glass surfaces. In this paper we propose to utilise the powder blasting process for miniaturised microsystems applications, like glass microchip devices for electrophoresis applications and inertial sensors of a few millimeters $[11,12]$. Inertial sensors are usually composed of silicon, but also piezo-electric materials like quartz are used. Fabrication is mostly by bulk wet etching or advanced plasma etching procedures. Depending on the used micropatterning processes, feature sizes range from a few millimeters down to a few micrometers. We show that micropowderblasting is also a competitive technology for these microsystems applications. Micropatterning resolution typically is around $50 \mu \mathrm{m}$ (for powder particle size of $30 \mu \mathrm{m}$ ) but can be smaller when smaller size erosion powders are used. The erosion rate is on the order of $1 \mathrm{~mm} / \mathrm{min}$, much higher than obtainable by wet and dry etching processes. Moreover, for inertial sensor applications, the silicon can be replaced by cheaper glass materials. In Section 2, we present our experimental set-up, consisting of a high-pressure nozzle and discuss the system used to measure the particle velocity. Subsequently, in Section 3 we discuss the influence of characteristic process parameters like pressure and nozzle-substrate distance on particle velocity. In addition, we discuss the surface morphology of the eroded glass surface and the influence of the pattern size on the erosion rate, as well as the influence of the angle of incidence on the eroding particle beam. In Section 4, we show a few applications, for which the powder blasting microfabrication represents an interesting option, like glass microchips for microfluidic applications, inertial sensors or the realisation of micropatterned hard magnetic particlebased composites. Finally, in Section 5, we present our conclusions.

\section{Experimental set-up}

All the erosion experiments are performed using a Texas Airsonics abrasive jet machine, type HP-2. The powder, consisting of $30 \mu \mathrm{m}$ size alumina particles $\left(\mathrm{Al}_{2} \mathrm{O}_{3}\right)$, is dosed by a vibration feeder and injected in a compressed air flux through a rubber tube to the nozzle. As represented in Fig. 1, the nozzle is mounted on a rounded support which permits easy modification of the incidence angle $\theta$ of the powder beam to the target by steps of $5^{\circ}$, from $0^{\circ}$ (normal incidence) up to $60^{\circ}$. The distance $d$ from the nozzle to the target can also be changed in a range from 4 to $8.5 \mathrm{~cm}$. We obtain a uniform substrate surface exposure, using an $x$-axis translation stage for the substrate and a $y$-axis translation stage for the nozzle. For masking certain glass areas against erosion, a laser-cut $0.5 \mathrm{~mm}$ thick metal mask containing the desired pattern is used. Pattern resolution using this process is on the order of few micrometers. The mask is in close contact with the glass due to the magnetic coupling with a hard magnet-based substrate table.

An essential parameter of the microparticle erosion process is the particle velocity. In order to determine the latter, we use a double disk system. A photograph of a double disk system is shown in Fig. 2. The two disks are parallel and separated by a $1.5 \mathrm{~cm}$ air gap. The upper disk has a $1 \mathrm{~mm}$ size hole permitting the particles to pass through in a very narrow beam. These particles are collected on a glass target fixed on the lower disk. The disk is rotated at rotation speeds between 3900 and 13,200 rpm. Each time, we compare the static impact profile (no disk rotation) on the glass target with the profiles corresponding

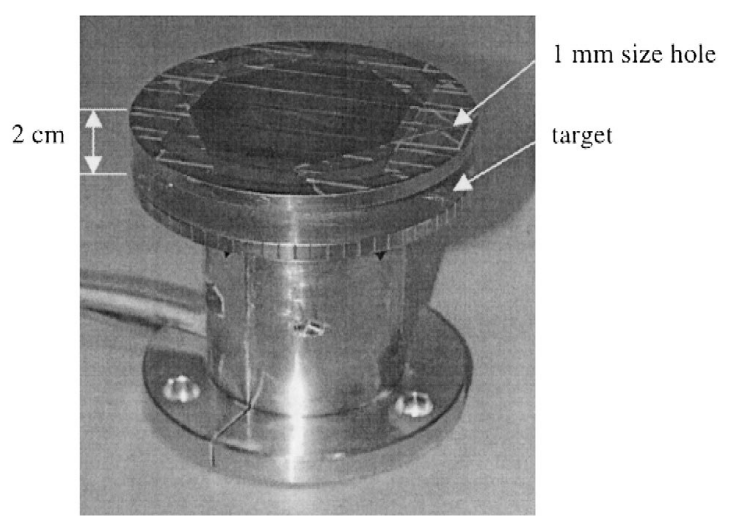

Fig. 2. Double-disk system used to determine the particle velocity. 


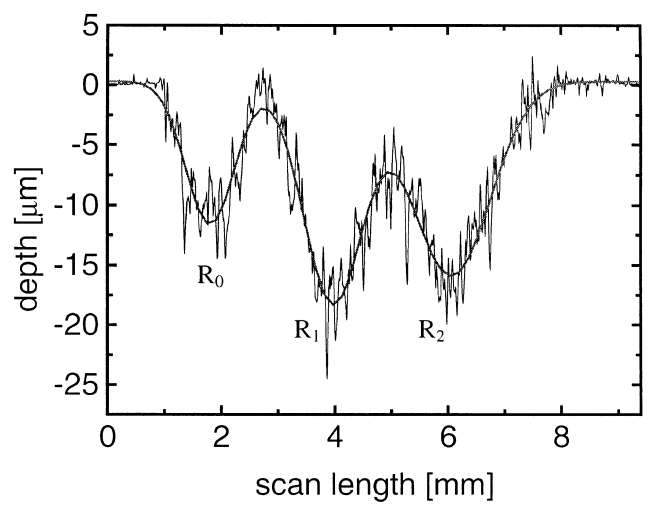

Fig. 3. Surface scan of a first glass sample mounted on the double disk system. Rotation speeds $R_{0}, R_{1}$ and $R_{2}$ are, respectively 0,6600 and $13,200 \mathrm{rpm}$, and the measured particle velocity is $191 \mathrm{~m} / \mathrm{s}$ for $p=0.3$ $\mathrm{MPa}$ and $d=4 \mathrm{~cm}$.

to two different disk rotation speeds. After the disk rotation experiment, we scan the erosion profiles of the glass using an Alpha-step 500 profilometer. Two examples of such scan are presented in Fig. 3. The first dip corresponds with a rotation speed $R=0 \mathrm{rpm}$, while the next two dips correspond to $R=6600$ and $13200 \mathrm{rpm}$, respectively. To have a profile of similar depth for the three dips, the $R=0$ rpm experiment is done during a much smaller exposure time than the true rotating disk experiments. One should note that it is not the actual depth of the dips that is important, but rather its lateral position on the glass substrate. The scan profiles look very noisy, as the material removed per impact is relatively large with respect to the total erosion depth. We fit the experimental curves using three Gaussians, the maximum of which gives the mean particle speed $v$ that we use for evaluation of our experiments and which is given by

$v=\frac{2 \pi r e R}{60 l}$

where $r$ is the disk radius, $e$ is the air gap between the two disks, $R$ is the rotation speed of the double-disk, and $l$ is the distance from one dynamic spot and the static one (see Fig. 3). We find a particle velocity $v=191 \mathrm{~m} / \mathrm{s}$ under the condition of the experiment (nozzle pressure $p=0.3 \mathrm{MPa}$ and $d=4 \mathrm{~cm}$ ).

\section{Experimental characterisation of the erosion process}

\subsection{Powder velocity and surface morphology}

We have performed multiple measurements of the powder velocity varying the applied pressure $p$ and the distance $d$ between the nozzle and the substrate. The influence of these two parameters on the particle velocity is shown in Figs. 4 and 5.

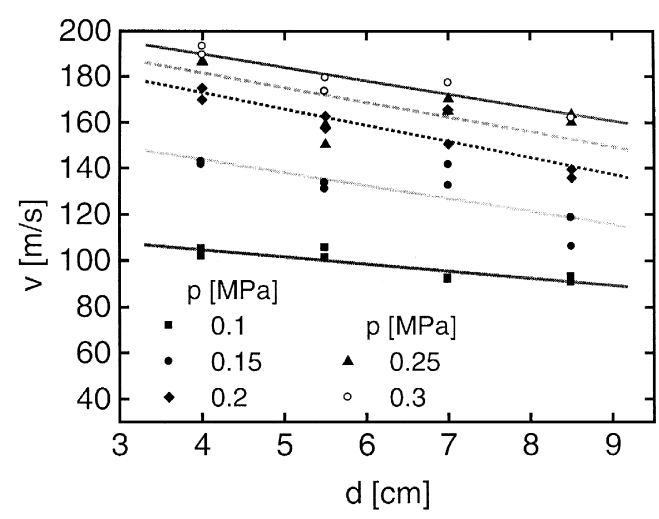

Fig. 4. Influence of the distance between the nozzle and the substrate on the particle velocity for different pressures $p$.

As can be seen from Fig. 4, the variation of the distance $d$ shows a decreasing particle velocity as a function of the pressure $p$ applied to the nozzle. In addition, the decrease is more significant for larger particle velocities. This behaviour can be understood using the velocity profile of an axisymmetric jet, which predicts a decrease in velocity $v$ with increasing distance from the jet nozzle. We also observe that, with increasing pressure $p$, the relative velocity enhancement tends to diminish due to the approach of the beam velocity to the sound velocity $(310 \mathrm{~m} / \mathrm{s})$, posing a limit to the maximum obtainable particle speed [13]. The same velocity data are represented in Fig. 5 against the applied pressure with the nozzle-target distance as a parameter, clearly showing the saturating behaviour for higher pressures. It clearly is the applied pressure, and not the nozzle-target distance, which is the determining factor in obtaining a certain particle velocity. The collection of data in Figs. 4 and 5 suggests a clear dependence of particle velocity on nozzle to substrate distance and applied nozzle pressure. However, it is also seen that individual points sometimes are not on the general trend curves. We believe the reason for this inaccuracy is the way in which particle

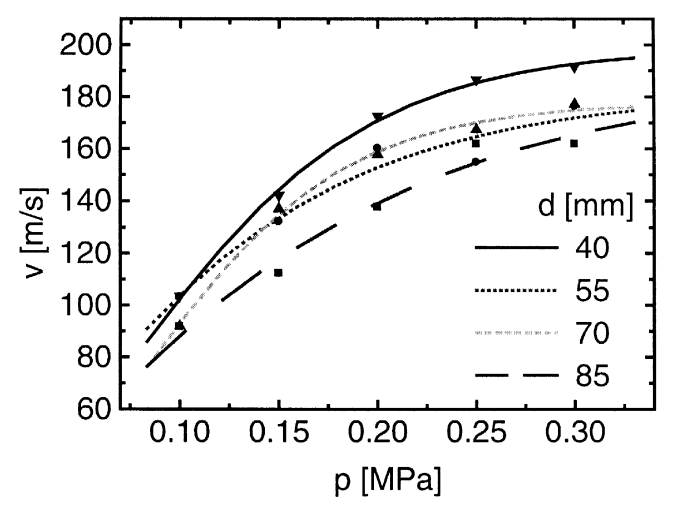

Fig. 5. Particle velocity as a function of pressure for different distances $d$ from the nozzle to the target. 
velocity is derived from erosion data, as shown in Fig. 3. Indeed, the determination of the exact position of the erosion depth minima can be influenced by the local surface roughness. From the particle velocity, one can also determine the kinetic energy $E_{\mathrm{k}}=(1 / 2) m v^{2}$ of a particle, where the average single particle mass $m=0.45 \mu \mathrm{g}$ for a $30-\mu \mathrm{m}$ alumina particle. Kinetic energy is not linear in $p$ but shows a saturation-like behaviour, associated with the approach of velocity of sound at higher nozzle pressures, as shown in Fig. 5.

The physics of the erosion process of sharp hard particles impacting on a brittle substrate is well known $[5,9,14,15]$. The impact of a single particle with a velocity $v$ on a substrate is schematically shown in Fig. 6. Increasing the nozzle pressure gives both a better erosion efficiency $E_{\text {eff }}$ and a higher etching rate $E_{\text {rate }}$. Fig. 7(a) is a scanning electron microscope (SEM) picture of a single particle indentation in a glass substrate, showing clearly the lateral cracks and the consequent material removal volume next to the line of original particle impact. Fig. 7(b) shows the effect of multiple impacts on a glass substrate. As we can see, the result of multiple single impacts gives rise to a significant roughness. This was already clear from the profile scans of Fig. 3, showing roughness amplitude of a few micrometers for $30-\mu \mathrm{m}$ particle size. The dependence of roughness on particle and target material parameters is also well understood and is described in detail in Ref. [6].

\subsection{Geometrical effects on erosion rate}

To characterise the erosion process and its functionality for microsystems fabrication, we investigate the dependence of the erosion rate on mask feature size. We use 0.5 $\mathrm{mm}$ thin steel mask with different square hole sizes, ranging in width from 100 to $3000 \mu \mathrm{m}$. The complete surface is exposed to a uniform particle flux and the resulting erosion depth of the various holes is measured using the focalisation depth of a standard optical microscope. Intrinsically, this method has nanometric accuracy.

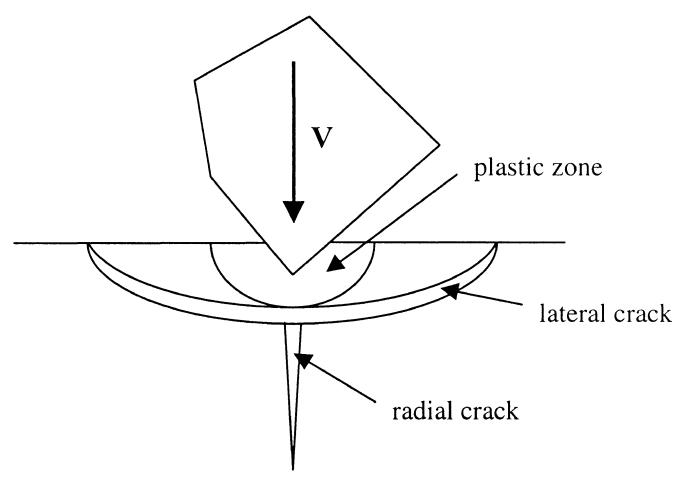

Fig. 6. Schematic view of the cracks generated by an indenting particle. (a)

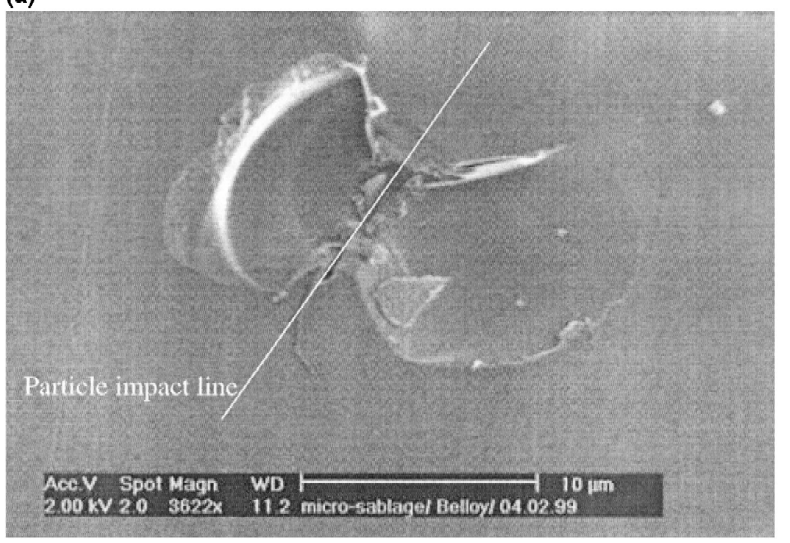

(b)

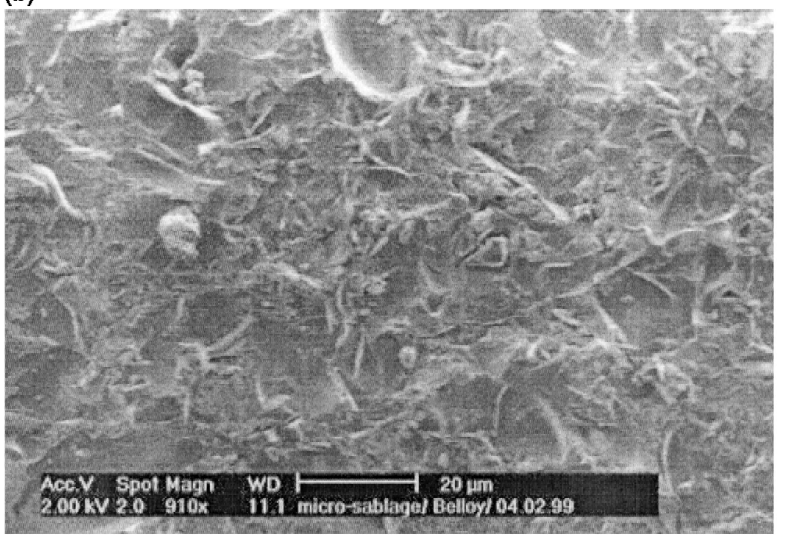

Fig. 7. (a) SEM graph of a single particle impact site on a glass wafer. (b) SEM picture of a powder-blasted glass surface.

Fig. 8 is a photograph of such test sample made from soda-lime glass. From left to right, one observes each time two identical holes with decreasing size. Measuring the total weight of the eroding particles used for etching a certain surface area, and comparing this with the weight of the material removed from the substrate, allows us to determine the erosion rate, as plotted in Fig. 9 for two different samples etched with the same experimental conditions, except the etching times which differ by a factor of 3.2. For mask opening sizes above $1.5 \mathrm{~mm}$ we observe a mask size independent etching depth. In the $0.5-1.0 \mathrm{~mm}$

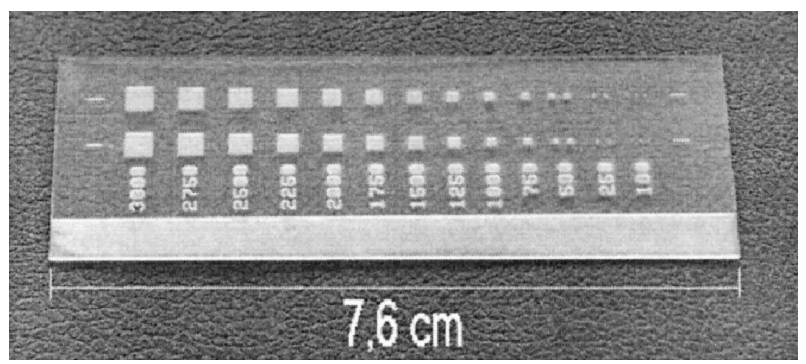

Fig. 8. Sandblasted glass sample with different mask pattern sizes. 


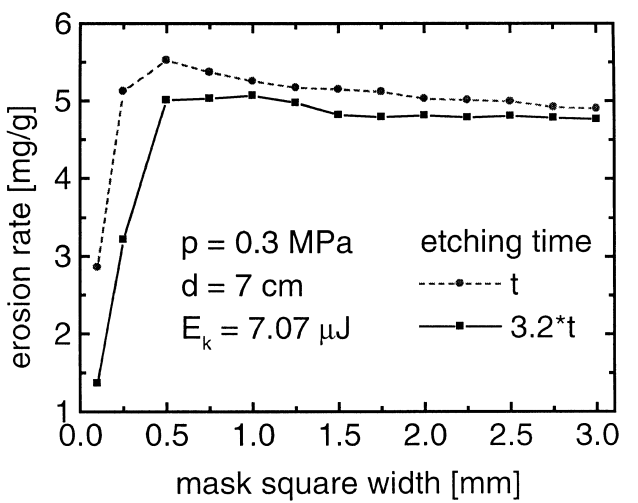

Fig. 9. Erosion rate as a function of the mask pattern size for two different etching times.

range there is a slight enhancement of the erosion depth, while the latter strongly decreases for still smaller mask feature sizes. The reason for the mask size dependent erosion depth is of geometrical origin. When the opening in the mask is too small with respect to the particle size, it would be very difficult for the particle to enter the hole and etch in depth. On the other hand, particle reflections occur at the side wall of the already etched structure, giving a slight overexposure of eroding particles near the structure edges [16]. Evidently, for a certain hole size, due to these secondary particle effects, there will be a deeper erosion at the centre of the structure, as we observe around mask sizes of $0.5-1.0 \mathrm{~mm}$. Clearly, the thickness of the mask also has an influence on the geometrical characteristics of the erosion profile. A thicker mask will diminish particle exposure within a hole, due to more mask side hole reflections of the particles and smaller kinetic energy being transferred to the substrate. The mask thickness has also an effect on the removal rate of used particles from within the hole. It is difficult to determine which of these two limiting factors is dominant.

We obtain a good consistency between the two etching times used and a principle agreement with erosion experiments of Refs. [6,7]. These curves can easily be transformed in erosion efficiency, since a single particle has an average weight of $0.45 \mu \mathrm{g}$ and a kinetic energy $E_{\mathrm{k}}=7.07$ $\mu \mathrm{J}$ for the given parameter settings. The erosion efficiencies are calculated to be $0.3 \mathrm{mg} / \mathrm{J}$, again in agreement with Refs. [6,7].

\section{Microsystems applications}

As said before, powder blasting technology has been brought up to a high technological level, thanks to the modern flat panel display applications [8-10]. Up to now, the technology has not been used for the realisation of microsystems or micromechanical structures. Here, we want to demonstrate a few possible applications. Fig. 10(a) and (b) are photographs of a microfluidic glass chip containing a cross-shaped microchannel of $100 \mu \mathrm{m}$ width and $10 \mu \mathrm{m}$ depth. A first glass wafer contains this eroded channel and is fusion-bonded with a second glass wafer containing the four access holes, also made using powder blasting [17]. Our glass bonding process gives a hermetically sealed channel with a surface structure, which is very suitable for the attachment of polymer gels for electrochromatographic applications. The total structure is shown in Fig. 10(a), while a detail of the cross channel is shown in Fig. 10(b). The observed channel wall roughness can pose limits on separation resolution of the microchannel, but it is beneficial for polymer bonding in electrochromatography.

A second application is the microstructuring of composite hard magnetic layers. These are composed of small magnetic $\mathrm{Sm}_{5}$ Co particles embedded in a hardened SU-8 epoxy matrix [18]. The liquid $\mathrm{Sm}_{5} \mathrm{Co}$ particle containing SU-8 solution first is spun onto a substrate and then hardened in a magnetic field for the orientation of the magnetic particles. This gives continuous layers of the hard magnet composite. Powder blasting proved to be an extremely interesting process for the realisation of hard magnet microstructures, to be utilised in magnetic sensing applications. A structural detail of a hole etched in only $2 \mathrm{~s}$

(a)

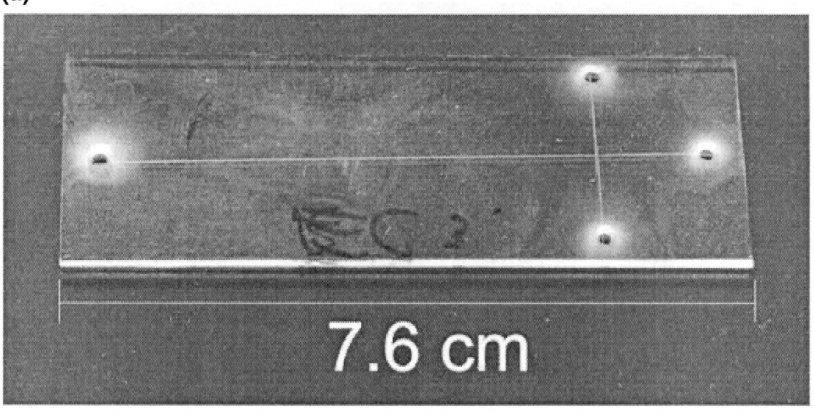

(b)

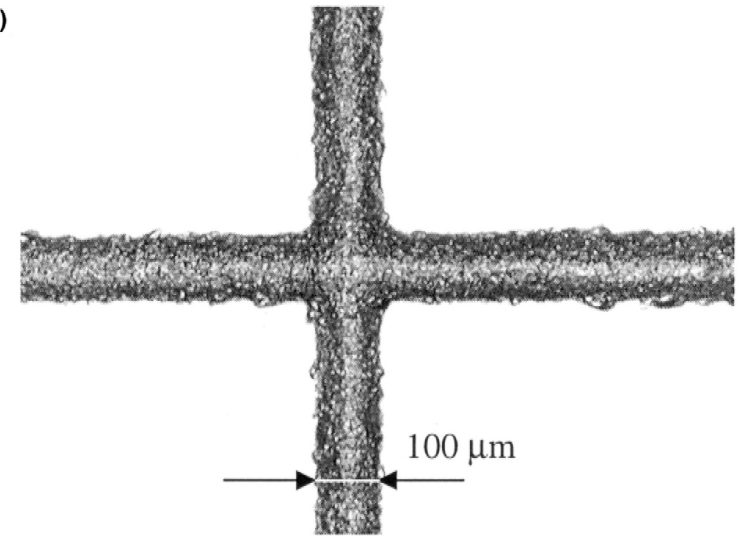

Fig. 10. (a) Holes and channels structured by sandblasting for microfluidic applications. (b) Cross channel microstructured by powder blasting in a glass substrate. 
in the composite hard magnetic layer of $100 \mu \mathrm{m}$ thickness is shown in Fig. 11. Also for this application, powder blasting is an extremely rapid and reliable microstructuration process.

Finally, we also propose to use powder-blasting technology for microelectromechanical (MEMS) applications. We have fabricated cantilever beams with various sensing masses in Pyrex glass wafers for inertial sensor applications. Fig. 12(a) shows such a structure made by a two-step erosion process: the first one consists in etching through the complete wafer, thereby defining the sensing mass and thick supporting beam; using a second mask, we have locally thinned the cantilever beam for tuning the resonance frequency of this device. Alternatively, such result could be obtained in one single step using the mask size depending erosion rate of the powder blasting process (see Fig. 9). We typically find resonance frequencies of 1500 $3000 \mathrm{~Hz}$ for these type of structures, depending on the geometrical dimensions. Another way to change the geometry of the mass supporting beam, and hence the resonance frequency, is to apply an oblique erosion process. For the device of Fig. 12(b) we have narrowed down the supporting beam by two oblique exposures at $45^{\circ}$.

We use two different measurement systems to characterise the realised accelerometer beams, by using alternatively (i) a digital camera connected to a monitor on which we measure the cantilever beam deflection, from which we deduce the resonance frequency, and (ii) a capacitance variation detection system constituted by the metallised beam mass and a fixed counter electrode, using an HP 4194A impedance gain/phase analyser. Fig. 13(a) shows experimental results of the cantilever beam deflection as a function of the applied frequency for a $4 \mathrm{~mm}$ long structure of the type shown in Fig. 12(a) with a mass of 4.87 $\mathrm{mg}$. We find a quality factor $Q=f_{\text {res }} / \Delta f$ of about 100 in air, where $f_{\text {res }}$ is the resonance frequency and $\Delta f$ the

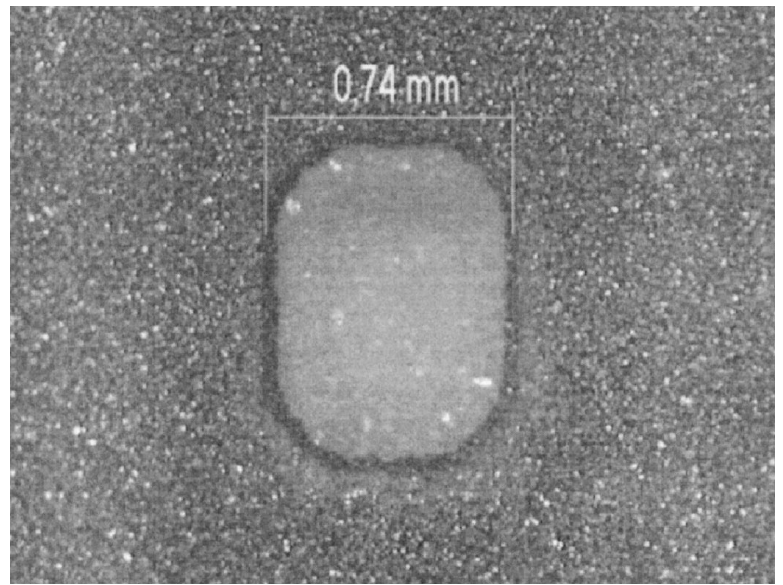

Fig. 11. Picture of a hole structured by sand blasting in a hard epoxy layer with embedded magnetic particles.

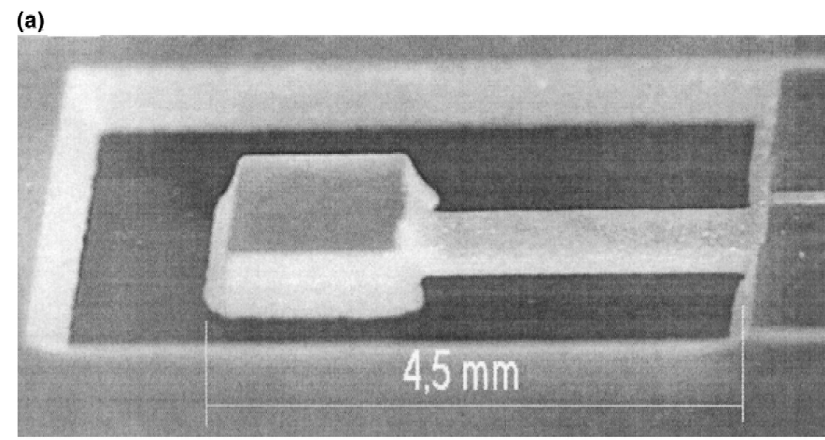

(b)

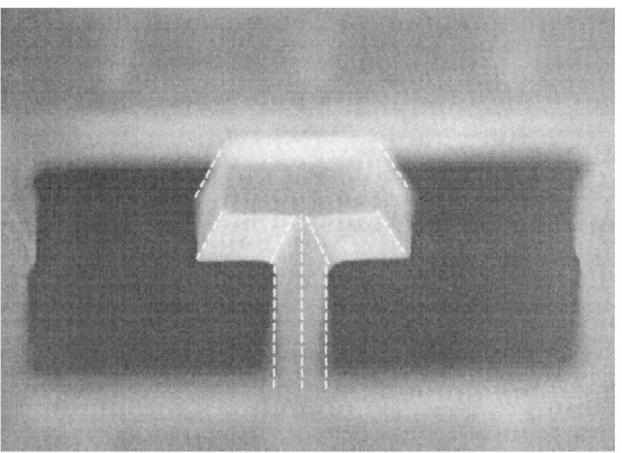

Fig. 12. (a) Picture of an accelerometer beam realised in two steps by powder blasting from the two substrate sides. (b) Glass cantilever beam realised by a two-step angular powder blasting process from one wafer side. The triangular shape of the cantilever beam is schematically indicated.

frequency width at half maximum of the resonance peak. Fig. 13(b) presents the calculated theoretical capacitance $C$

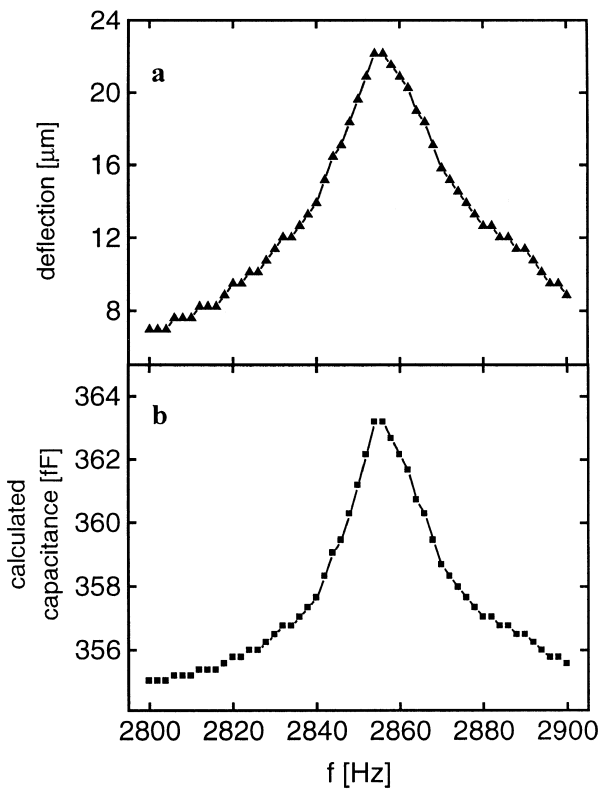

Fig. 13. (a) Deflection of the accelerometer beam as a function of the applied excitation frequency. (b) Capacitance, calculated using Eq. 2 with as input the experimentally determined detection amplitude $b$, as a function of applied frequency. 


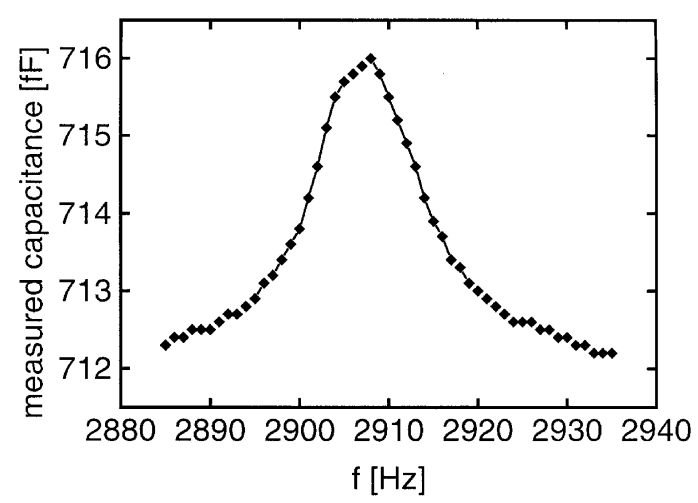

Fig. 14. Directly measured capacitance using an impedance analyser.

associated with the measured deflection amplitude $b$ of Fig. 13(a) by the relation:

$C=\frac{1}{\pi} \int_{0}^{\pi} \frac{\varepsilon_{0} S}{a+b \cos \alpha} \mathrm{d} \alpha=\frac{\varepsilon_{0} S}{\sqrt{a^{2}-b^{2}}}$

where $S$ is the capacitance surface and $a$ the static gap between the electrode on the cantilever mass and its counter electrode.

With the impedance analyser, we also measure directly the capacitance of the same device, as presented in Fig. 14. There is a good qualitative agreement between Figs. 13(b) and 14. However, the measured capacitance values are a factor of 2 higher than the calculated values. We attribute this to the presence of parasitic capacitance in the measurement set-up, as device capacitance value is fairly low and therefore susceptible to parasitic effects. We also observe that the experimental capacitance variation $\Delta C$ over the frequency range studied is a factor of 2 smaller than the calculated one. This can be explained by the increased damping action in the narrow air-filled channel of the experimental capacitance measurement, while for the measurement of the vibration amplitude $b$ the cantilever is allowed to vibrate freely in air. The presence of air obviously is the explanation for the relatively low quality factor and capacitive sensitivity of $5.3 \mathrm{aF} / \mathrm{g}$ of the device. Another difference is the resonance frequency of Figs. 13(b) and 14. The difference of about $50 \mathrm{~Hz}$ can be explained by different clamping conditions of the cantilever support system during the vibration amplitude measurements and the direct capacitance measurement.

\section{Conclusions}

We have presented powder blasting technology as a new interesting option for microsystems fabrication. We have used a pressurised nozzle for directing abrasive $30-\mu \mathrm{m}$ $\mathrm{Al}_{2} \mathrm{O}_{3}$ particles to a glass substrate covered by a metal mask. We examined the basic parameters of the powder blasting erosion process as well as mask-related geometrical influences on the erosion rate. We have presented three possible applications for powder blasting in the field of microsystems: (i) the realisation of microfluidic chips for biochemical separations, (ii) the micropatterning of composite hard magnetic layers for mechatronic and magnetic sensor applications, and (iii) the realisation of inertial sensors in glass. Finally, we have presented the first mechanical and electrical characterisation of this new type of accelerometer structures.

\section{Acknowledgements}

We would like to thank Philips Research for financial and technical support for this research and especially P.J. Slikkerveer, P. Bouten and F. de Haas for useful discussions.

\section{References}

[1] Abstract book of the International Conference on Erosive and Abrasive Wear, Cambridge, 13th-17th September 1998, University of Cambridge.

[2] I.M. Hutchings, N.H. Macmillan, D.G. Rickerby, Further studies of the oblique impact of a hard sphere against a ductile solid, Int. J. Mech. Sci. 23 (1981) 639-646.

[3] M. Dündar, O.T. Inal, Erosion by Small Solid Particles, Abstract no. 66, International Conference on Erosive and Abrasive Wear, Cambridge, 13th-17th September 1998, University of Cambridge, 1998.

[4] G.P. Tilly, W. Sage, The interaction of particle and material behaviour in erosion processes, Wear 16 (1970) 117-165.

[5] M. Buijs, Erosion of glass as modeled by indentation theory, J. Am. Ceram. Soc. 77 (1994) 1676-1678.

[6] P.J. Slikkerveer, P.C.P. Bouten, F.H. in't Veld, H. Scholten, Erosion and damage by sharp particles, Wear 217 (1998) 237-250.

[7] M.A. Geltink-Verspui, Modelling abrasive processes of glass, $\mathrm{PhD}$ Thesis, Eindhoven University of Technology, 1998.

[8] T.S. Baller, G.G.P. van Gorkom, N. Lambert, E.A. Montie, P.H.F. Trompenaars, S.T. de Zwart, Construction and physical processing of Zeus panels, Philips J. Res. 50 (1996) 463-474.

[9] H.J. Ligthart, P.J. Slikkerveer, F.H. in't Veld, P.H.W. Swinkels, M.H. Zonneveld, Glass and glass machining in Zeus panels, Philips J. Res. 50 (1996) 475-499.

[10] T. Hirose, K. Kariya, M. Wakitani, A. Otsuka, T. Shinoda, Performance features of a 42-inch diagonal color plasma display, Fujitsu webpage information (www.fujitsumicro.com) 1997.

[11] D.J. Harrison, A. van den Berg (Eds.), Micro Total Analysis Systems '98, Proceedings of the $\mu$ Tas '98 Workshop, Banff, Canada, Kluwer Academic Publishers, Netherlands, 1998.

[12] Digest of Technical Papers, Transducers '99, The 10th International Conference on Solid-State Sensors and Actuators, Sendai, Japan, 1999, pp. 1500 and following.

[13] H. Tennekes, J.L. Lumney, A First Course in Turbulence, MIT Press, Cambridge, MA, 1972.

[14] D.B. Marshall, B.R. Lawn, A.G. Evans, Elastic/plastic indentation damage in ceramics: the lateral crack system, J. Am. Ceram. Soc. 65 (11) (1982) 561-566.

[15] I.M. Hutchings, Transitions, threshold effects and erosion maps, Key Eng. Mater. 71 (1992) 75-92.

[16] P.J. Slikkerveer, H. in't Veld, N. Verbeek, K. Valkering, Modelling of Patterned Erosive Etching by Hard Sharp Particles, Abstract no. 31, International Conference on Erosive and Abrasive Wear, Cambridge, 13th-17th September, University of Cambridge, 1998. 
[17] A. Sayah, D. Solignac, T. Cueni and M.A.M. Gijs, Development of novel low-temperature bonding technologies for microchip chemical analysis applications, Sens. Actuators, A, in press.

[18] B. Dutoit, P.A. Besse, H. Blanchard, L. Guérin, R.S. Popovic, High performance micromachined $\mathrm{Sm}_{2} \mathrm{Co}_{17}$ polymer bonded magnets, Sens. Actuators, A (1999) in press.

\section{Biographies}

Eric Belloy was born in Geneva, Switzerland, in 1972. He received his diploma in microengineering in 1997 from the Swiss Federal Institute of Technology of Lausanne (EPFL). His diploma work was the study, realisation and characterisation of microtips on $\mathrm{Si}$ and glass substrates, in use for the detection of the electrical activity of nerves cells. Since April 1997, he has worked as research assistant in the Institute of Microsystems at EPFL. He first developed the technology process for a new magnetic sensor based on the Hall effect. Then, he developed a new technology based on flex-foil patterning and assembling, in order to realise magnetic devices. His actual research area is on dry-etching by microsandblasting.

Sébastien Thurre and Edith Walckiers are graduate students in microengineering, who contributed to the present work during the course of a student research project.

Abdeljalil Sayah received his degree in physics in 1988 from University Mohammed V, Rabat, specialising in state solid physics. He obtained his AESA and DEA in microelectronics specialising in the technology of the components in 1990 and 1992, respectively. From 1993 until 1996 he worked as a research assistant in the CNET-Bagneux (Paris) Laboratory where he received a $\mathrm{PhD}$ degree for the thesis entitled "Realisation of silicon-based dielectrics optical waveguide on InP by photochemical deposition". In 1996, he joined the Applied Optics Institute (IOA) of the Swiss Federal Institute of Technology in Lausanne (EPFL) as a postdoctoral research. His research interests included the study and characterisation of optical fiber tips for scanning near field optical microscopy by protection layer liquid-phase etching and participated on the characterisation of Bragg grating produced in optical fibers by UV laser. He is now with the Institute for Microsystems (IMS) at EPFL. His current research interests are in inertial sensor (gyroscope), bio-sensors and fabrication technologies.
Martin A.M. Gijs received his degree in physics in 1981 from the Katholieke Universiteit Leuven, Belgium and his $\mathrm{PhD}$ degree in physics at the same university in 1986. He joined the Philips Research Laboratories in Eindhoven, The Netherlands, in 1987. Subsequently, he has worked there on micro- and nano-fabrication processes of high critical temperature superconducting Josephson and tunnel junctions, the microfabrication of microstructures in magnetic multilayers showing the giant magnetoresistance effect, the design and realisation of miniaturised motors for hard disk applications and the design and realisation of planar transformers for miniaturised power applications. He joined the Swiss Federal Institute of Technology of Lausanne (Ecole Polytechnique Féderale de Lausanne) in 1997 as a professor in the Institute of Microsystems of the Microengineering Department, where he is responsible for the Microsystems Technology group. His main interests are in developing technologies for novel inductive-type devices, new microfabrication technologies for microsystems fabrication in general and the development and use of microsystems technologies for biomedical applications in particular. 